\section{Mid-term outcomes of endovascular treatment for symptomatic chronic mesenteric ischemia}

\author{
Eva Schönefeld, Susanne Szesny, \\ Konstantinos P. Donas, \\ Georgios A. Pitoulias, \\ Giovanni Torsello
}

Centre of Vascular and Endovascular Surgery, University Hospital Münster, Germany

\section{Abstract}

The authors would present the mid-term outcomes with the use of stent-supported angioplasty in the treatment of symptomatic chronic mesenteric ischemia (CMI). The present study is a retrospective analysis of 36 patients undergoing endovascular treatment of symptomatic CMI, between November 2000 and September 2009. Primary study endpoints were defined as primary patency, periprocedural and midterm mortality and complications, and symptom improvement after intervention. Forty-one mesenteric arteries (77.3\% stenotic and $22.7 \%$ occluded vessels) were treated in 36 patients with 42 stents. In 30 patients $(83.3 \%)$ one visceral artery and in 6 cases (16.7\%) two visceral arteries were treated. Overall mortality was $16.7 \%(n=6)$ after a 60-month follow-up (mean follow-up period 30.1 months). Two early (<30-day) deaths were caused by visceral ischemia $(\mathrm{n}=2: 5.5 \%)$. Late death was procedure-related in one patient with re-occlusion of the superior mesenteric artery after 12 months. The other 3 patients died from non procedure-related causes; e.g. twice myocardial infarction. Initial symptom relief was observed in 29 patients (80.5\%); 7 patients reported no change. Primary patency was $83.3 \%$ after 5 years and secondary patency was $90.5 \%$ (38 out of 42 stents) among all patients. Two conversions to open surgery were documented. First-line endovascular approach of CMI is a reasonable strategy. Close follow-up is mandatory due to symptom recurrence and restenosis.

\section{Introduction}

Endovascular treatment for chronic mesenteric ischemia (CMI) is growing in popularity because of its lower periprocedural morbidity and mortality (4\%) than open surgery (9-18\%). Short-term outcomes are excellent, while longterm patency results of mesenteric stenting are still scarce. There are also little data to suggest that primary stenting is better than angioplasty alone, but the deployment of a stent may be valuable as a rescue procedure following dissection, vascular recoil, or thrombosis during angioplasty. Open surgery is the treatment of choice if multiple-vessel disease or ostial occlusion has to be treated in low-risk patients. Patients with severe comorbidities, such as coronary artery disease, may take advantage from endovascular procedure. Aim of the present study is to present and compare midterm outcomes after use of stent-supported angioplasty in the treatment of symptomatic CMI.

\section{Materials and Methods}

In a retrospective analysis 36 patients with CMI underwent between November 2000 and September 200942 endovascular interventions. $77.3 \%$ of the treated lesions were stenotic and $22.7 \%$ occlusive. Patients data and comorbidities were documented. Coronary artery disease (CAD) was defined as any history of myocardial infarction, angina, congestive heart disease or coronary intervention. Renal disease was defined as a baseline serum creatinine level of $>1.5 \mathrm{mg} / \mathrm{dL}$. In the collective 22 patients (61.1\%) suffered from CAD and 11 patients $(30.5 \%)$ had elevated creatinine levels. Additively, all mesenteric stenotic lesions were demonstrated by duplex ultrasound and angiography. Follow-up protocol included patients' records, clinical visits, duplex ultrasound and biplanar x-ray imaging examinations before discharge, 6 months later, and then yearly.

\section{Study endpoints}

Primary study endpoints were defined as primary patency, periprocedural and mid-term mortality and morbidity, as well as symptom improvement after endovascular therapy.

Primary patency was considered failed when patients developed in-stent restenosis/occlusion. Primary patency was defined as a lack of recurrent clinical symptoms and lack of vessel occlusion. Restenosis $<70 \%$ and freedom of symptoms were documented but left without therapeutical intervention. Patients whose recurrences were treated with an open surgical revascularization were censored as failures of primary patency. If duplex scan velocity remained increased, according to a stenosis $>70 \%$, angiogram was performed.

Secondary patency was defined as vessel patency after secondary procedures as thrombectomy, bypass grafting or angioplasty.

Periprocedural morbidity and mortality were defined as adverse events occurring within the first 30 days after endovascular intervention or during initial hospitalization. Major morbidity
Correspondence: Eva Schönefeld, University Hospital Münster, Waldeyerstrasse 30, 48149 Münster, Germany.

Tel. +49.2518351717 - Fax: +49.2518345787.

E-mail: eva.schoenefeld@ukmuenster.de

Key words: chronic mesenteric ischemia, endovascular therapy, revascularization.

Conflict of interest: the authors report no conflicts of interest.

Received for publication: 11 April 2011.

Revision received: 3 June 2011.

Accepted for publication: 3 June 2011.

This work is licensed under a Creative Commons Attribution 3.0 License (by-nc 3.0).

(C) Copyright E. Schönefeld et al., 2011

Licensee PAGEPress, Italy

Surgical Techniques Development 2011; 1:e2

doi:10.4081/std.2011.e2

included any occurrence resulting in prolonged hospital stay: bowel infarction, myocardial infarction, stroke, acute renal failure, respiratory failure, or multi-organ failure (MOF). Vascular access complications, e.g. pseudoaneurysm, that required operative revision were also documented and reported. Symptom improvement after intervention was observed and documented as symptom relief. Overall survival or death from any cause was determined by hospital records and follow-up. CMIrelated survival reported deaths that were consecutive to primary intervention (PTA/stent) or subsequent mesenteric ischemia. Statistical analysis was performed to describe and determine relevant risk factors and treatment mortality and morbidity. Here Fisher's exact test or Pearson's Chi-Square test were performed for categorical variables. ANOVA and t-test were used to test for differences in continuous variables. Kaplan-Meier method was used to assess survival rate and primary patency. All statistical data were performed using a statistical software program (SPSS 18.0 ${ }^{\mathrm{TM}}$, SPSS Inc., Chicago, IL, USA). Values were expressed as mean \pm SD (standard deviation). Statistical significance was accepted with a $P$ value of less than 0.05 .

\section{Procedural data}

All mesenteric interventions were performed in angiographic suite or endovascular hybrid operating room with fixed imaging systems. Interventions were performed under local anaesthesia via a brachial $(n=25 ; 69.4 \%)$ or femoral $(\mathrm{n}=11 ; 30.5 \%)$ approach. Access site selection was based on specific anatomy of each patient as well as surgeons preferences. Periprocedural heparin bolus 5000 IU i.v. was given in all cases. After biplanar angiographic 
imaging was performed angled selective catheters were used to engage the vessel ostium. Through a $6 \mathrm{~F}$ sheaths lesions were crossed by hydrophilic guidewires. After angioplasty with a non-compliant balloon appropriately sized balloon-expandable stents were deployed. If the ostium appeared stenotic, stents were positioned to protrude slightly in the aortic lumen. Self-expanding stents were used for non-orificial lesions. In the majority of the cases the Palmaz Genesis Stent was used (Cordis Corporation ${ }^{\mathrm{TM}}$, Miami, FL, USA) $(\mathrm{n}=22 ; 61.1 \%)$. In the remaining cases an Herkulink Stent (Abbott Vascular ${ }^{\mathrm{TM}}$, Santa Clara, CA, USA) was implanted in nine patients (25\%), a SMART Control ${ }^{\mathrm{TM}}$ (Cordis Corporation Europe, Waterloo, Belgium) in one, a Carotid Wall Stent ${ }^{\mathrm{TM}}$ (Boston Scientific, Natick, MA, USA) in one or an XPert Stent ${ }^{\mathrm{TM}}$ (Abbott Vascular ${ }^{\mathrm{TM}}$ ) in one.

Distal embolic protection systems or atherectomy devices were not used in the collective. Clopidogrel was given for 8-12 weeks in addition to lifelong low-dose aspirine once a day.

\section{Results}

Technical success rate was $100 \%$. Thirty patients had a single-vessel intervention (83.3\%), and $6(16.6 \%)$ a two-vessel treatment. Stent dislocations, migrations or fractures were not observed. Every patient had at least one of the following clinical symptoms: postprandial abdominal pain $(n=15 / 40.5 \%)$, significant weight loss (>15\%kg) ( $\mathrm{n}=16 / 43.2 \%)$, food fear $(n=2 / 5.4 \%)$, nausea $(n=5 / 13.5 \%)$, and diarrhea ( $n=12 / 32.4 \%)$ along with ultrasound and angiographic evidence of a significant stenosis $(\geq 70 \%)$ of $\geq 1$ mesenteric artery. Indication for endovascular intervention inhabited minimally one clinical symptom and a lack of different cause of disease. Patient characteristics are shown in Table 1 . Initial symptom relief was achieved in 29 patients (80.5\%); 7 patients reported no change. In all patients food fear disappeared after revascularization, and body weight increased.

\section{Morbidity and mortality}

Overall procedural morbidity and mortality is given in Table 2. Postoperative complications included 2 bowel ischemic events, one stroke and 2 myocardial infarctions. In one patient pseudo-aneurysm at the access site had to be repaired.

Mortality included two early deaths (day 1 and 15 after intervention) caused by visceral ischemia $(n=2 ; 5.6 \%)$. Overall mortality for any cause was $16.7 \%$ (6 patients) after a mean follow-up of 30.1 months.

Later on one procedure-related death occurred after conversion an open procedure. His death was caused by multiple organ failure. Unrelated mortality beyond 30 days of follow-up was $8.8 \%$ (3 out of 34 patients). Overall survival rate is given in Figure 1 (83.3\%).

\section{Patency}

The 5-year primary and secondary patency in the collective of 36 patients and 42 stent deployments was $83.3 \%$ and $90.5 \%$ (Figure 2). Univariate analysis showed no specific risk factor for restenosis affecting primary patency. The mean interval of restenosis was $25.4 \pm 18.9$ months.

\section{Discussion}

The current study aims to evaluate outcomes after endovascular treatment of visceral artery lesions in patients with chronic mesenteric ischemia. Overall mortality was $16.7 \%$ (6 patients) after mean follow-up of 30.1 months. Two patients died within the first 30 days (day 1 and 15 after intervention) caused by visceral ischemia $(\mathrm{n}=2 ; 5.6 \%)$. No patient was lost to follow-up. 5-year primary and secondary patency in the collective of 36 patients and 42 stent deployments was $83.3 \%$ and $90.5 \%$.

Peck et al. ${ }^{1-4}$ published in 2010 their results in a comparable collective of 49 patients with 66 endovascular treated visceral arteries. Primary patency was $63.9 \%$ after 48 months with a restenosis rate of $28.6 \%$ requiring rein-
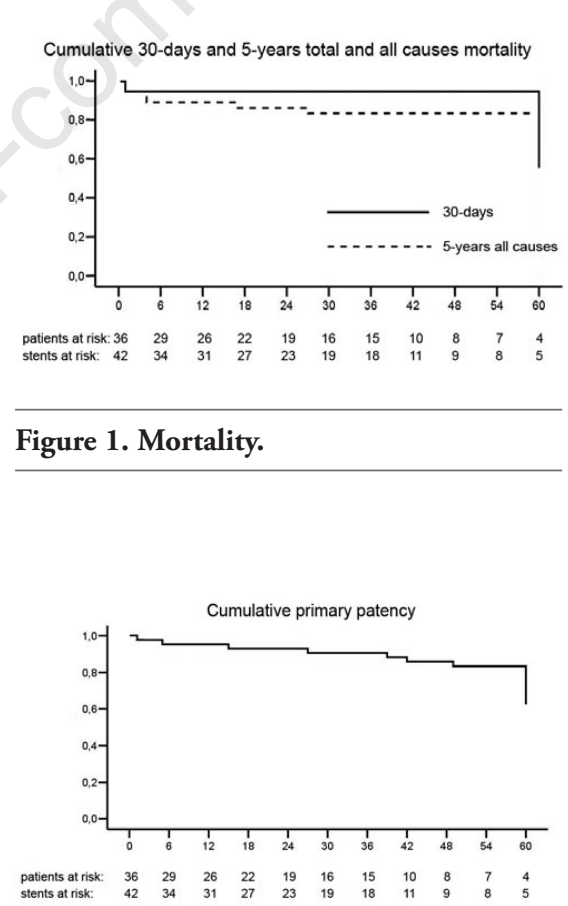

Figure 2. Patency of the stents. tervention. In our series primary patency was over $90 \%$, and open surgery was necessary in one case. Two deaths were CMI-related.

In his overview Sreenarasimhaiah ${ }^{4}$ emphasized that technical success is dependent on anatomical features, such as short mesenteric stenosis, non-ostial occurrence and one-vessel involvement. Mortality rates in several articles vary from $0-17 \%$ after endovascular therapy. ${ }^{5-7}$ Morbidity rate ranges between $19-54 \%{ }^{4}$ (Table 3 ). A cumulative analysis of Kougias et al. in $2007^{8,9}$ showed technical success of endovascular treatment in $91 \%$ of the cases, with a complication rate of $9 \%$, and a 30 -day mortality of $3 \%$. Restenosis rate was comparable to the presented collective with $26 \%$.

Clinical and technical success of endovascu-

Table 1. Patients characteristics.

\begin{tabular}{lcc} 
& $\mathrm{n}=$ & $\%$ \\
Age (years) & $68.0 \pm 9.7$ & \\
Gender (m/f) & $20 / 16$ & $56.8 / 43.2$ \\
\hline CMI symptoms (yes/no) & $36 / 0$ & 100 \\
$\quad$ Postprandial pain & 15 & 40.5 \\
Weight loss & 16 & 43.2 \\
Food fear & 2 & 5.4 \\
$\quad$ Nausea & 5 & 13.5 \\
Diarrhea & 12 & 32.4 \\
Tobacco use (current/ex) & $10 / 6$ & $29.7 / 16.2$ \\
\hline Hypertension & 28 & 77.7 \\
Coronary artery disease & 10 & 27.0 \\
\hline Arrhythmia & 2 & 5.4 \\
Diabetes & 10 & 27.0 \\
\hline Hyperlipemia & 12 & 32.4 \\
Renal insufficiency & 9 & 24.3 \\
\hline COPD & 7 & 18.9 \\
Stroke/TIA & 5 & 13.5 \\
\hline Prior bowel surgery & 7 & 21.6 \\
Pancreatitis & 2 & 5.4 \\
\hline
\end{tabular}

Table 2. Mortality and morbidity during 60 months of follow-up $(n=36)$.

\begin{tabular}{lcc} 
Event & n & $\%$ \\
Early mortality & 2 & 5.6 \\
Procedure-related mortality & 3 & 8.3 \\
\hline Late mortality & 4 & 11.1 \\
Total mortality & 6 & 16.7 \\
\hline Major morbidity & 6 & 16 \\
$\quad$ Bowel ischemia & 2 & 5.6 \\
$\quad$ Stroke & 1 & 2.9 \\
Acute renal failure & 0 & 0 \\
$\quad$ Cardial decompensation & 2 & 5.6 \\
Infection & 0 & 0 \\
$\quad$ Neoplasm & 1 & 2.9 \\
Access repair & 1 & 2.9 \\
(pseudoaneurysm/occlusion) & & \\
Interval of restenosis & $25.4 \pm 18.9$ \\
(mean \pm SD in months) & \multicolumn{2}{l}{} \\
\hline
\end{tabular}


Table 3. Literature review: endovascular approach to chronic mesenteric ischemia.

\begin{tabular}{lcccccc} 
Authors & n/vessels & $\begin{array}{c}\text { Technical } \\
\text { success }\end{array}$ & $\begin{array}{c}\text { Mortality } \\
\%\end{array}$ & $\begin{array}{c}\text { Morbidity } \\
\%\end{array}$ & $\begin{array}{c}\text { Restenosis } \\
\%\end{array}$ & $\begin{array}{c}\text { Primary } \\
\text { patency }\end{array}$ \\
Kougias et al. (2007) & 328 & 91 & 3 & 9 & 28 & $\mathrm{n} / \mathrm{r}$ \\
Kougias et al. (2009) & 58 & $\mathrm{n} / \mathrm{r}$ & 21 & 21 & 71 & 82 \\
\hline Schermerhorn et al. (2009) & 6342 & $\mathrm{n} / \mathrm{r}$ & 8 & 20.2 & $\mathrm{n} / \mathrm{r}$ & $\mathrm{n} / \mathrm{r}$ \\
Peck et al. (2010) & 66 & 100 & 18.9 & 16.3 & 28.6 & 63.9 \\
\hline Oderich et al. (2009) & 146 & 100 & 2.7 & 37 & 8 & 88 \\
Gibbons et al. (2010) & 328 & 91 & 4 & 9 & 28 & 65 \\
\hline Sreenarasimhaiah (2005) & $\mathrm{V}$ & 95 & $0-17$ & $19-54$ & 13 & 88 \\
Sarc et al. (2008) & 87 & 90 & 11 & 30.8 & 37 & 69 \\
\hline
\end{tabular}

$\mathrm{n} / \mathrm{r}$, not reported; $\mathrm{V}$, variable authors/review.

lar therapy in the visceral segment may also depend on endovascular experience of each interventionalist. ${ }^{10-13}$ The presented study showed content results for learning curve on endovascular interventions already started in 1996/1997. Schermerhorn et al. ${ }^{11}$ analyzed the largest collective in 2009. They collected all data about mesenteric revascularization in the United States from 1988 until 2006. Totally, 6342 endovascular procedures were reported with an increasing amount of percutaneous interventions with lower morbidity and mortality after minimally invasive procedure compared to bypass surgery. Complications, e.g. bowel necrosis and consecutive resection, were more common after bypass surgery than after PTA or/and stenting.

A publication was written by Kougias et al. in 20099. Comparison of endovascular versus surgical procedure was given in a smaller collective. Overall morbidity after endovascular intervention was lower than after open procedure $(\mathrm{P}=0.01)^{9}$ and mortality was similar.

In conclusion, treatment selection is most important and the endovascular therapy has already its indication. ${ }^{14-17}$ Endovascular intervention is a safe alternative to open surgical bypass. ${ }^{14,18}$ Clinical effectiveness increases to comparable levels. High-risk patients profit from endovascular approach, but re-occlusion is, even in more than one treated vessel, more frequent. Nevertheless patients' hospitalization is shorter after endovascular intervention. ${ }^{12,16,18}$

There were some limitations of the study. At first, it was a retrospective evaluation. There was no control data collective, e.g. comparison to open intervention. In the Vascular Surgery department reported, first line endovascular approach is standard regimen.

\section{Conclusions}

First-line endovascular approach of CMI is a reasonable strategy. Primary patency rate of $83.3 \%$ was convincing to the concept. A time interval with a higher risk of restenosis could not be found nor any prevalent risk factors to reveal CMI. Close follow-up is mandatory for the detection of restenosis. Especially for patients with severe comorbidities endovascular means play an important role.

\section{References}

1. PeckMA, Conrad MF, Kwolek CJ, et al. Intermediate-term outcomes of endovascular treatment for symptomatic chronic mesenteric ischemia. J Vasc Surg 2010;51: 140-7.e1-2.

2. Acosta S. Epidemiology of mesenteric vascular disease: clinical implications. Semin Vasc Surg 2010;23:4-8.

3. Chandra A, Quinones-Baldrich WJ. Chronic mesenteric ischemia: how to select patients for invasive treatment. Semin Vasc Surg 2010;23:21-8.

4. Sreenarasimhaiah J. Chronic mesenteric ischemia. Best Pract Res Clin Gastroenterol 2005;19:283-95.

5. Resch TA, Acosta S, Sonesson B. Endovascular techniques in acute arterial mesenteric ischemia. Semin Vasc Surg 2010;23:29-35.

6. Sarc TP, Altinel 0, Kashyap V, et al. Endovascular treatment of stenotic and occluded visceral arteries for chronic mesenteric ischemia. J Vasc Surg 2008;47: 485-91.
7. Ke SR, Chiang FT. Endovascular therapy for a patient with chronic mesenteric ischemia. J Formos Med Assoc 2010;109: 315-9.

8. Kougias P, El Sayed H, Zhou W, Lin PH. Management of chronic mesenteric ischemia. the role of endovascular therapy. J Endovasc Ther 2007;14:395-405.

9. Kougias P, Huynh TT, Lin PH. Clinical outcomes of mesenteric artery stenting versus surgical revascularization in chronic mesenteric ischemia. Int Angiol 2009;28: 132-7.

10. Oderich GS, Gloviczki P, Bower TC. Open surgical treatment for chronic mesenteric ischemia in the endovascular era: when it is necessary and what is the preferred technique? Semin Vasc Surg 2010;3:36-46.

11. Schermerhorn ML, Giles KA, Hamdan AD, et al. Mesenteric revascularization: management and outcomes in the United States, 1988-2006. J Vasc Surg 2009;50: 341-8.e1.

12. Oderich GS, Bower TC, Sullivan TM, et al. Open versus endovascular revascularization for chronic mesenteric ischemia: riskstratified outcomes. J Vasc Surg 2009;49: 1472-9.e3.

13. Gibbons CP, Roberts DE. Endovascular treatment of chronic arterial mesenteric ischemia: a changing perspective? Semin Vasc Surg 2010;23:47-53.

14. Björck M, Wanhainen A. Nonocclusive mesenteric hypoperfusion syndromes: recognition and treatment. Semin Vasc Surg 2010;23:54-64.

15. Park WM, Cherry KJ Jr, Chua HK, et al. Current results of open revascularization for chronic mesenteric ischemia: a standard for comparison. J Vasc Surg 2002;35: 853-9.

16. Debus ES, Luther B, Daum H, LarenaAvellaneda A. Chronische intestinale Ischämie. [Chronic intestinal ischemia]. Chirurg 2009;80:473-84.

17. Matsumoto AH, Angle JF, Spinosa DJ, et al. Percutaneous transluminal angioplasty and stenting in the treatment of chronic mesenteric ischemia: results and longterm followup. J Am Coll Surg 2002; 194(1 Suppl):S22-S31.

18. Assar AN, Abilez OJ, Zarins CK. Outcome of open versus endovascular revascularization for chronic mesenteric ischemia: review of comparative studies. J Cardiovasc Surg (Torino) 2009;50:509-14. 